\title{
Novel Microtomy Methods For Small, Hard And Precious Extraterrestrial And Terrestrial Rocky Materials
}

\author{
D.E. Brownlee ${ }^{1}$ and D. Joswiak ${ }^{1}$ \\ 1. Dept of Astronomy, Univ. of Washington, Seattle, WA, USA
}

The use of Focused Ion Beam (FIB) methods has provided revolutionary capabilities for making thin-sections for TEM, ion probe and beam-line microanalyses of extraterrestrial materials. Although FIB methods have been a remarkable enabling-technology in the study of extraterrestrial materials [1,2], the production of thin-sections by diamond knife ultramicrotomy of hard rocky materials [3] still plays important roles. One advantage of microtomy is that it can efficiently, inexpensively and quickly provide large numbers of slices from small precious samples. For example, a single $5 \mu \mathrm{m}$ diameter particle can be cut into as many as one hundred $50 \mathrm{~nm}$ serial sections, ideally preserving all of a complex sample. Adjacent slices can be distributed to different investigators and analyzed by different microbeam analytical methods. This capability is particularly important for complex, valuable samples such as small particles returned to Earth by comet and asteroid sample return missions and also for small complex samples such as presolar grains found in meteorites and interplanetary dust. The ability to use adjacent slices provides the capability to do coordinated isotopic mapping with the ion probe and detailed TEM analyses of submicron components. Similar coordination can also be done with slices and the remaining bulk sample (potted butt) and this is invaluable for certain analyses such as precision isotopic analyses that require greater sample thickness than normal FIB or microtome sections provide. Additional benefits of microtomy are the lack of radiation damage or beam deposition effects that can occur in FIB samples. We will describe three microtomy methods that we use for cutting small hard extraterrestrial samples and mounting them on TEM grids.

Intact sections of hard materials: When brittle materials such as silicate minerals are cut with diamond knives, they fracture because of the sharp bend at the knife-edge and $>$ micron brittle components often fracture into a ordered fabric of elongated micron-sized shards. TEM analyses of microtomed rocky materials are usually done on shards and some shards experience substantial movement that disturbs original textural relations. In some cases, shards are displaced beyond the original particle perimeter. Shard formation in hard phases cannot be prevented, even with a $35^{\circ}$ diamond knife, but we have developed a simple method that keeps the shards in their original positions and the sample sections remain intact, resembling the craquelure (crazing crack texture) on old paintings. This process is done by putting a thin plastic film on the surface of the sample block just before each slice is cut and then dissolving the film off afterwards. Small samples are mounted in acrylic or epoxy bullets that are trimmed so that the cut-face is a $100 \mu \mathrm{m} \times 200 \mu \mathrm{m}$ trapezoid mesa with near vertical sides about $30 \mu \mathrm{m}$ high. Usually the mesa contains a single 3-10 $\mu \mathrm{m}$ sample. Just before each cut is made, we manually touch the mesa with the tip of a millimeter-sized triangular piece of filter paper that has just been dipped in a $0.1 \%$ solution of collodion in amyl acetate. The microtome is stopped and the coating is done under microscopic observation and left to dry, all in only a few 
seconds time. The collodion film is later removed by dissolution with condensed chloroform vapor in a special sub-boiling grid-washing apparatus. The entire process is actually very simple, it works extremely well and provides excellent sections even of hard silicate minerals such as olivine that are at least as large as $50 \mu \mathrm{m}$. The sections are excellent for microanalysis but the shards, while preserved in place, are tilted relative to each other and this affects large-scale HAADF and conventional imaging where thickness or crystal orientation contributes to image contrast. FIB sections provide better textural information on sizes larger that a few microns, but this microtome method provides generally excellent slices of hard materials that are often difficult or impossible to section by conventional microtomy. Although we have not tried it, it likely that this method could be used on samples that are much larger that can be sectioned with the FIB.

Alignment: A major issue with microtomy of small priceless samples has always been the challenge of aligning individual or serially sectioned samples in the center of grid square or bars that hold carbon support films. One method that we have found to be very effective is to pick sections off the microtome boat with a loop (the Diatome Perfect Loop) and then use a kolinksy sable hair to force the sections to their desired location as the water dries on the grid. We pick up the slices with the loop, then pick up a carboncoated grid and place the loop holder on a movable magnetic clamp to center the suspended loop under a stereomicroscope. We then use a fine sable hair mounted on a needle, controlled by a simple glide-plate micromanipulator, to press down on the section or sections so that they can be aligned as the water evaporates. When the water has dried to only a few microns thick, the section can be precisely positioned above the grid support film, before the water evaporates. We speed up the drying process using filter paper wicks and by heating by a focused $10 \mathrm{w}$ halogen lamp that also plays a critical role in illuminating floating sections that can be $<50 \mathrm{~nm}$ thick and sometimes difficult to see.

Concentrating submicron particles: A unique role of microtomy is the ability to make sections of large arrays of micron-sized particles all mounted on a flat plane. This might have many applications but we developed the "Rubber Stamp" method to find rare submicron presolar grains that are contained in primitive interplanetary dust particles. A single $10 \mu \mathrm{m}$ dust particle is a loose aggregate that might contain $10^{5}$ submicron grains, only a tiny fraction of which are presolar. The particle can be gently broken down into its diverse components and dispersed over a wide flat area. With the rubber stamp we concentrate component grains onto a $50 \mu \mathrm{m}$ square flat silicone rubber mesa, formed by casting clear silicone rubber into a flat-bottomed $50 \mu \mathrm{m}$ hole. Under a stereo microscope, the rubber mesa is repeatedly pushed against the dispersed grains. Particles adhere to the rubber analogous to using tape to remove cat hair from a shirt. When the desired density of particles is obtained, the mesa with its monolayer of particles is embedded in Weld-On 40 acrylic or epoxy and sectioned. With proper alignment, the result is sections of large numbers of spatially concentrated micron and smaller particles all lying in the same plane. This method could also be used to concentrate very rare small components that are chemically isolated from a larger sample and left in a dispersed state on a flat surface.

[1] R. Stroud, Cometary Dust in Astrophysics (2003), p6011.

[2] G. Graham et al. Meteoritics and Planetary Science 43 (2008), p561.

[3] J. Bradley and D. Brownlee, Science 231 (1986), p1542. 